\title{
Commentary: Emergence of a Stable Cortical Map for Neuroprosthetic Control
}

\author{
Mikhail A. Lebedev* \\ Department of Neurobiology, Duke University, Durham, NC, United States
}

Keywords: brain-machine interfaces, plasticity, linear models, fixed decoder, preferred direction

\section{A commentary on}

\section{Emergence of a Stable Cortical Map for Neuroprosthetic Control}

by Ganguly, K., and Carmena, J. M. (2009). PLoS Biol. 7:e1000153. doi: 10.1371/journal.pbio. 1000153

This highly cited paper by Ganguly and Carmena (2009) reported a case of neuroplasticity associated with the operation of a brain-machine interface (BMI). Neuroplasticity is of great interest to BMI developers because of its causal role in the embodiment of neural prostheses (Lebedev and Nicolelis, 2006; Dobkin, 2007; Koralek et al., 2012; Shenoy and Carmena, 2014; Kraus et al., 2016; Gulati et al., 2017).

Ganguly and Carmena reported that small populations of neurons (from 10 to 15) recorded in monkey primary motor cortex (M1) adapted to operating a BMI based on a fixed linear decoder. The decoder was trained once and left unchanged for several weeks. The population activity

OPEN ACCESS

Edited by:

Peter Brunner,

Albany Medical College, United States

Reviewed by:

Karunesh Ganguly,

University of California, San Francisco,

United States

${ }^{*}$ Correspondence:

Mikhail A. Lebedev mikhail.a.lebedev@gmail.com

Specialty section:

This article was submitted to Neuroprosthetics,

a section of the journal

Frontiers in Neuroscience

Received: 21 May 2017 Accepted: 06 November 2017 Published: 24 November 2017

Citation:

Lebedev MA (2017) Commentary: Emergence of a Stable Cortical Map

for Neuroprosthetic Control.

Front. Neurosci. 11:642.

doi: 10.3389/fnins.2017.00642 patterns underwent plastic modifications and stabilized on an optimal "cortical map" that assured accurate performance of center-out movements with a screen cursor. Moreover, monkeys learned to operate shuffled decoders, where the original neuronal weights were randomly reassigned.

Here I comment on three issues arising from this paper: (1) the proper way to assess neuronal tuning under BMI control; (2) the constraints imposed on neuronal tuning properties by a fixed decoder; and (3) the problem of measuring changes in tuning when both neuronal activity and cursor trajectories change.

\section{NEURONAL TUNING UNDER BMI CONTROL}

Classically, neuronal directional tuning is the dependence of neuronal firing rate on the direction of arm reaching movement (Georgopoulos et al., 1982; Schwartz et al., 1988). Typically, an M1 neuron exhibits the highest firing rate when the arm moves in the direction, called preferred direction (PD). If the arm moves at an angle with respect to the $\mathrm{PD}$, firing rate declines proportionally to the cosine of the angle.

While it is tempting to use a directional tuning analysis for BMI control (Taylor et al., 2002; Lebedev et al., 2005; Ganguly and Carmena, 2009), the results of such an analysis should be interpreted with caution. The main caveat here (and this is rarely explained in the literature) is the strong dependence of neuronal tuning characteristics on the decoder parameters. Taking a singletap positional decoder (Georgopoulos et al., 1983, 1986; Taylor et al., 2002; Schwartz et al., 2004) as an example, the relationship between the firing rate of a given neuron, $N(t)$, and cursor coordinates, $x$ and $y$, is expressed by the equations:

$$
\begin{aligned}
& x=a N(t)+\text { contribution_of_other_neurons } \\
& y=b N(t)+\text { contribution_of_other_neurons }
\end{aligned}
$$


Let's assume first that the correlation is very low between $N(t)$ and the activity of other neurons. In this case, contribution_of_other_neurons does not interfere with the neuronal directional tuning, and the vector $[a, b]$ defines the neuron's PD. This PD would persist during BMI control even if the neuron produces nonsensical firing unrelated to motor commands or feedback from the cursor.

Next, if the correlation of $N(t)$ with the activity of the other neurons is substantial, the neuron's PD may be different from $[a$, $b$ ]. Consider the case of two neurons with positively correlated rates:

$$
\begin{aligned}
& x=a_{1} N 1(t)+a_{2} N 2(t) \\
& y=b_{1} N 1(t)+b_{2} N 2(t) \\
& N 2(t)=K N 1(t)+\text { noise }
\end{aligned}
$$

In this case, the PDs of neurons 1 and 2 are $\left[a_{1}+K a_{2}, b_{1}\right.$ $\left.+K b_{2}\right]$ and $\left[a_{1} / K+a_{2}, b_{1} / K+b_{2}\right]$, respectively, i.e., neurons affect each other's directional tuning. Again, these PDs would be produced even if the neuronal firing is nonsensical. Furthermore, if the contribution of one neuron, for example neuron 2 , is much stronger than the contribution of the other, both have the same PD, $\left[a_{2}, b_{2}\right]$. Such "capture" of the PD by the stronger weighted neurons may explain the previously reported similarity of many neurons' PDs during BMI control (Carmena et al., 2003; Lebedev et al., 2005; Green and Kalaska, 2011; O’Doherty et al., 2011).

These simple considerations are relevant to the previous studies that reported changes in PD during BMI control (Taylor et al., 2002; Green and Kalaska, 2011), including the fixeddecoder study of Ganguly and Carmena, where a linear decoder generated cursor position from the activity of a small population of M1 neurons. Ganguly's and Carmena's decoder extracted joint angles instead of $x$ and $y$ coordinates (Figures 1A,B), but for simplicity a linear approximation can be used:

$$
X(t)=b+\sum_{u, i} a_{u i} N_{i}(t-u)
$$

where $X(t)$ is the BMI output ( $x, y$ and/or their time derivatives), $b$ is zero intercept, $i$ is neuron number, $u$ is Wiener filter tap, $a_{u \mathrm{i}}$ are regression weights, and $N_{i}$ are neuronal rates. This equation differs from Equation (1) by the presence of tap structure in the representation of neuronal rates. Ganguly and Carmena used ten 100-ms taps.

Ganguly and Carmena assessed directional tuning by measuring neuronal rates in two windows: a short, 200-ms, window, and a long, 2s, window. The former was used "for the analysis of the directional modulation of the firing rate with respect to the actual direction of cursor movements" ${ }^{1}$ and the latter for "for calculating the mean firing rate versus target direction". None of these analyses was designed to capture the 10tap structure of the decoder. Furthermore, Ganguly and Carmena

\footnotetext{
${ }^{1}$ The description of the 200-ms window analysis is not entirely clear in Ganguly and Carmena. To the best of my understanding, only the first $200 \mathrm{~ms}$ following movement onset were analyzed, and neuronal activity during this period was compared to the actual direction of cursor movement, not target direction.
}

did not report the values of $a_{u i}$, so at this point it is impossible to assess the PDs incorporated in the decoder.

Although Ganguly and Carmena did not analyze PD for each tap, one previous study (Lebedev et al., 2005) conducted such an analysis for three conditions: manual cursor control with a joystick, BMI control assisted by the joystick, and BMI control without arm movements. It was found that neuronal PDs rotated with incrementing taps for the first two conditions but not for the third.

\section{CAN NEURONAL TUNING CHANGE FOR A FIXED DECODER?}

The main claim of Ganguly and Carmena is that M1 ensemble starts with one pattern of directional tuning, and then gradually adapts to a new, more stable pattern as the monkey perfects the BMI control (Figure 1D). Similar changes in PD had been previously reported by Taylor and her colleagues for an adaptive decoder (Taylor et al., 2002). But is this even possible if the decoder is fixed and its weights define a basic PD structure? The answer is "yes" because, as explained above, changes in correlation between the neurons (Equation 2) could result in PD modifications. Drifts in neuronal firing rates could explain PD changes, as well. According to Ganguly and Carmena, mean rate increased in 8 of 15 neurons in one monkey, and 6 of 10 in the other. These changes in mean rates could reconfigure the joint angles (Figure 1A), which could in turn affect the neuronal PDs measured with respect to linear displacements (Equation 3).

\section{ASSESSMENT OF CHANGES IN NEURONAL TUNING WHEN CURSOR PATTERNS CHANGE}

While the monkeys in the experiments of Ganguly and Carmena clearly improved their performance (Figure 1C), an overt strategy (e.g., pressing on the arm restraint in different directions) cannot be ruled out. The authors reported that they "concurrently performed video and surface electromyogram (EMG) recordings from proximal muscle groups" but did not present any results that would convince that directionally tuned EMG modulations did not occur. The same authors reported a paradigm with a better control for overt strategies (Ganguly et al., 2011), but the task was different; monkeys possibly used directionally tuned preparatory activity previously reported for instructed-delay tasks (Weinrich and Wise, 1982) to drive the cursor in that study.

With or without an overt strategy, cursor trajectories changed very dramatically from the very convoluted ones during the initial training days to nearly straight lines during late in training (Figure 1C). The presence of such dramatic changes makes inadequate the analysis of PDs using the wide 2-s window that covered a convoluted trajectory in the beginning of the training and a straight trajectory in the end. The 200-ms window analysis does not appear adequate either. Indeed, cursor velocity was generated from ten 100-ms taps (Equation 3, Figure 1B). The 200-ms window represents only $20 \%$ of this neuronal 


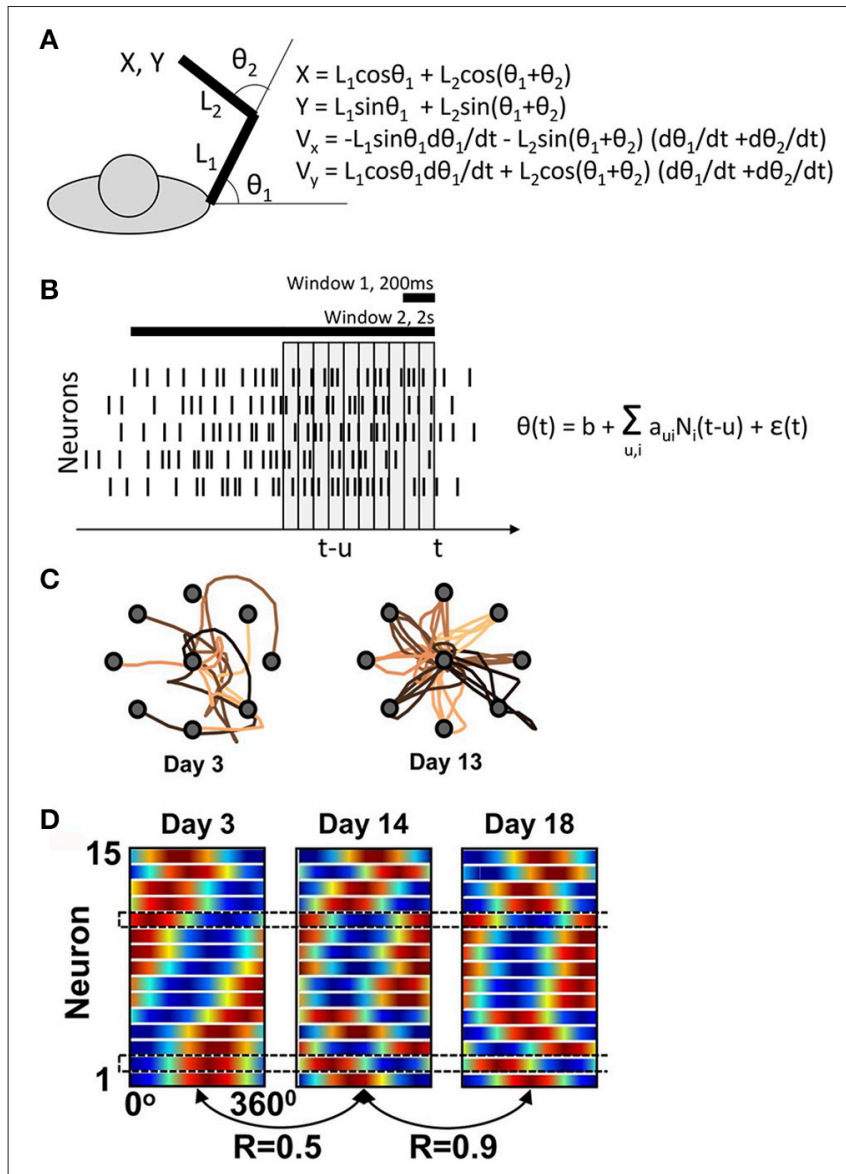

FIGURE 1 | Ganguly and Carmena's experiment. (A) Task schematics. The behavioral apparatus suspended the arm in a horizontal plain. Monkeys were free to flex and extend their shoulder and elbow to control the cursor coordinates, defined by the center of the hand. On the right, the equations are shown for the translation of joint angles into the cursor Cartesian coordinates. (B) Schematics of the BMl decoder, which was composed of two Wiener filters converting neuronal activity into the shoulder and elbow angles. The decoders contained ten 100-ms taps and were trained on the manual performance in a center-out task. For BMl control, the monkeys' arms were isometrically restrained. Horizontal bars illustrate the time windows (200 ms and 2s) that Ganguly and Carmena used in their directional tuning analyses. None of these analyses matched the tap structure of the decoder. (C) Examples of cursor trajectories on different training days. (D) Directional tuning analysis using a 200-ms window representing the initial portion of cursor movement from the screen center to the target. Directional tuning characteristics for different neurons (horizontal lines) are represented by sinusoids fitted to neuronal data. The color plots are normalized to have the same minimal and maximal values for all neurons. With this representation, tuning depths cannot be compared across neurons or different training days. (C) and (D) are adapted from Ganguly and Carmena (2009).

contribution and also does not capture the complexity of rate changes with different taps. Clearly, a much more complex procedure was used to generate cursor movements from neuronal activity compared to the attempt to measure how the generated movements depended on the activity of individual neurons.

There is no easy solution for assessing neuronal tuning in a reliable way when cursor patterns dramatically change, but several analyses could be helpful. First, PDs could be measured for each tap individually (Lebedev et al., 2005) and compared with the tap-dependent structure of PDs (also called impulse response function) enforced by the decoder. This analysis may show a pattern of PD rotations like the one observed during manual control, meaning that the monkey learned to generate neuronal patterns matching the decoder design. Alternatively, PDs would stay the same across the taps, and this would indicate that the monkey cannot produce neuronal patterns matching the training data and uses some other strategy. The tap-dependent PD patterns could be then compared to the corresponding shapes of cursor trajectories for different training days. Additionally, since correlated neuronal activity can affect PDs during BMI control (Equation 2), changes in correlation between the neurons should be assessed, as well. Finally, a simpler one-tap decoder could be used to minimize the number of factors affecting neuronal PDs. Most importantly, PD should be treated as a parameter highly dependent on the decoder settings (Equations 1,2) rather than a separate property of brain activity. Without such analyses, the claim that a monkey learned a fixed decoder would not be sufficiently substantiated.

\section{CONCLUSIONS}

Ganguly and Carmena's study is very convincing regarding monkey ability to learn new types of BMI control with a fixed decoder, but less convincing regarding the neuronal mechanisms underlying this learning. Some of the questionable issues could be resolved using data analyses that match more closely the decoder structure. Yet, more experiments or analysis of previously collected data may be needed with easily tractable BMI algorithms [e.g., liner decoder with just one tap (Taylor et al., 2002)] to clarify neuronal adaptations under constraints imposed by a fixed decoder.

Since the publication of Ganguly's and Carmena's work, a dynamical-systems perspective (as opposed to representation perspective) gained popularity (Shenoy et al., 2013). This new view emphasizes changes in neuronal patterns in a multidimensional neuronal space and downplays the importance of the description in terms of neuronal-tuning parameters, such as PD. Among the findings that emerged from this approach is the discovery of neuronal subspaces that correspond to different types of neuronal processing (Kaufman et al., 2014; Lebedev, 2017). Moreover, it has been demonstrated that plastic adaptations related to BMI operations occur more readily if the BMI control signal is derived from particular, action-potent subspaces (Sadtler et al., 2014), the result that can be interpreted as a constraint on Ganguly's and Carmena's adaptive "cortical maps". Yet, even with these new developments neuronal plasticity during BMI control remains a largely unexplored problem that needs proper methods for quantification. Particularly, "cortical maps" should be distinguished from the "map" applied by the decoder.

\section{AUTHOR CONTRIBUTIONS}

The author confirms being the sole contributor of this work and approved it for publication. 


\section{REFERENCES}

Carmena, J. M., Lebedev, M. A., Crist, R. E., O’Doherty, J. E., Santucci, D. M., Dimitrov, D. F., et al. (2003). Learning to control a brainmachine interface for reaching and grasping by primates. PLoS Biol. 1:e42. doi: 10.1371/journal.pbio.0000042

Dobkin, B. H. (2007). Brain-computer interface technology as a tool to augment plasticity and outcomes for neurological rehabilitation. J. Physiol. 579, 637-642. doi: 10.1113/jphysiol.2006.123067

Ganguly, K., and Carmena, J. M. (2009). Emergence of a stable cortical map for neuroprosthetic control. PLoS Biol. 7:e1000153. doi: 10.1371/journal.pbio.1000153

Ganguly, K., Dimitrov, D. F., Wallis, J. D., and Carmena, J. M. (2011). Reversible large-scale modification of cortical networks during neuroprosthetic control. Nat. Neurosci. 14, 662-667. doi: 10.1038/nn.2797

Georgopoulos, A. P., Caminiti, R., Kalaska, J. F., and Massey, J. T. (1983). Spatial coding of movement: a hypothesis concerning the coding of movement direction by motor cortical populations. Exp. Brain Res. Suppl. 7:336. doi: 10.1007/978-3-642-68915-4_34

Georgopoulos, A. P., Kalaska, J. F., Caminiti, R., and Massey, J. T. (1982). On the relations between the direction of two-dimensional arm movements and cell discharge in primate motor cortex. J. Neurosci. 2, 1527-1537.

Georgopoulos, A. P., Schwartz, A. B., and Kettner, R. E. (1986). Neuronal population coding of movement direction. Science 1416-1419. doi: 10.1126/science.3749885

Green, A. M., and Kalaska, J. F. (2011). Learning to move machines with the mind. Trends Neurosci. 34, 61-75. doi: 10.1016/j.tins.2010.11.003

Gulati, T., Guo, L., Ramanathan, D. S., Bodepudi, A., and Ganguly, K. (2017). Neural reactivations during sleep determine network credit assignment. Nature 20, 1277-1284. doi: 10.1038/nn.4601

Kaufman, M. T., Churchland, M. M., Ryu, S. I., and Shenoy, K. V. (2014). Cortical activity in the null space: permitting preparation without movement. Nat. Neurosci. 17, 440-448. doi: 10.1038/nn.3643

Koralek, A. C., Jin, X., Long, II., J. D., Costa, R. M., and Carmena, J. M. (2012). Corticostriatal plasticity is necessary for learning intentional neuroprosthetic skills. Nature 483, 331-335. doi: 10.1038/nature10845

Kraus, D., Naros, G., Bauer, R., Leão, M. T., Ziemann, U., and Gharabaghi, A. (2016). Brain-robot interface driven plasticity: distributed modulation of corticospinal excitability. Neuroimage 125, 522-532. doi: 10.1016/j.neuroimage.2015.09.074

Lebedev, M. (2017). Commentary: Cortical activity in the null space: permitting preparation without movement. Front. Neurosci. 11:502. doi: 10.3389/fnins.2017.00502
Lebedev, M. A., Carmena, J. M., O’Doherty, J. E., Zacksenhouse, M., Henriquez, C. S., Principe, J. C., et al. (2005). Cortical ensemble adaptation to represent velocity of an artificial actuator controlled by a brain-machine interface. J. Neurosci. 25, 4681-4693. doi: 10.1523/JNEUROSCI.4088-0 4.2005

Lebedev, M. A., and Nicolelis, M. A. (2006). Brain-machine interfaces: past, present and future. Trends Neurosci. 29, 536-546. doi: 10.1016/j.tins.2006.07.004

O’Doherty, J. E., Lebedev, M. A., Ifft, P. J., Zhuang, K. Z., Shokur, S., Bleuler, H., et al. (2011). Active tactile exploration using a brain-machine-brain interface. Nature 479, 228-231. doi: 10.1038/nature10489

Sadtler, P. T., Quick, K. M., Golub, M. D., Chase, S. M., Ryu, S. I., TylerKabara, E. C., et al. (2014). Neural constraints on learning. Nature 512:423. doi: $10.1038 /$ nature 13665

Schwartz, A. B., Kettner, R. E., and Georgopoulos, A. P. (1988). Primate motor cortex and free arm movements to visual targets in three-dimensional space. I. Relations between single cell discharge and direction of movement. J. Neurosci. 8, 2913-2927.

Schwartz, A. B., Moran, D. W., and Reina, G. A. (2004). Differential representation of perception and action in the frontal cortex. Science 303, 380-383. doi: $10.1126 /$ science. 1087788

Shenoy, K. V., and Carmena, J. M. (2014). Combining decoder design and neural adaptation in brain-machine interfaces. Neuron 84, 665-680. doi: 10.1016/j.neuron.2014.08.038

Shenoy, K. V., Sahani, M., and Churchland, M. M. (2013). Cortical control of arm movements: a dynamical systems perspective. Annu. Rev. Neurosci. 36, 337-359. doi: 10.1146/annurev-neuro-062111-150509

Taylor, D. M., Tillery, S. I., and Schwartz, A. B. (2002). Direct cortical control of 3D neuroprosthetic devices. Science 296, 1829-1832. doi: 10.1126/science. 1070291

Weinrich, M., and Wise, S. P. (1982). The premotor cortex of the monkey. J. Neurosci. 2, 1329-1345.

Conflict of Interest Statement: The author declares that the research was conducted in the absence of any commercial or financial relationships that could be construed as a potential conflict of interest.

Copyright (C) 2017 Lebedev. This is an open-access article distributed under the terms of the Creative Commons Attribution License (CC BY). The use, distribution or reproduction in other forums is permitted, provided the original author (s) or licensor are credited and that the original publication in this journal is cited, in accordance with accepted academic practice. No use, distribution or reproduction is permitted which does not comply with these terms. 